\title{
Sex differences in the traumatic stress response: PTSD symptoms in women recapitulated in female rats
}

\author{
Apryl E. Pooley ${ }^{1 *}$ (D), Rebecca C. Benjamin ${ }^{1}$, Susheela Sreedhar ${ }^{1}$, Andrew L. Eagle ${ }^{1,2}$, Alfred J. Robison ${ }^{1,2}$,
} Michelle S. Mazei-Robison ${ }^{1,2}$, S. Marc Breedlove ${ }^{1}$ and Cynthia L. Jordan ${ }^{1}$

\begin{abstract}
Background: Post-traumatic stress disorder (PTSD) affects men and women differently. Not only are women twice as likely as men to develop PTSD, they experience different symptoms and comorbidities associated with PTSD. Yet the dearth of preclinical research on females leaves a notable gap in understanding the underlying neuropathology of this sex difference.

Methods: Using two standard measures of PTSD-like responses in rats, the acoustic startle response (ASR) and dexamethasone suppression test (DST), we tested the effects of traumatic stress in adult male and female rats using two rodent models of PTSD, single prolonged stress and predator exposure. We then examined the neural correlates underlying these responses with cFos and glucocorticoid receptor immunohistochemistry in brain regions implicated in the traumatic stress response.

Results: We now report that adult male and female rats across two models of PTSD show consistent sex-specific responses that recapitulate fundamental differences of PTSD in men and women. Trauma-exposed males showed the well-established hyper-responsive phenotype of enhanced ASR and exaggerated negative feedback control of the hypothalamic-pituitary-adrenal axis, while the same traumatic event had little effect on these same measures in females. Dramatic sex differences in how trauma affected cFos and glucocorticoid receptor expression in the brain lend further support to the idea that the trauma response of male and female rats is fundamentally different.

Conclusions: Two standard measures, ASR and DST, might suggest that females are resilient to the effects of traumatic stress, but other measures make it clear that females are not resilient, but simply respond differently to trauma. The next important question to answer is why. We conclude that males and females show fundamentally different responses to trauma that do not simply reflect differences in resilience. The divergent effects of trauma in the brains of males and females begin to shed light on the neurobiological underpinnings of these sex differences, paving the way for improved diagnostics and therapeutics that effectively treat both men and women.
\end{abstract}

Keywords: Sex differences, Single prolonged stress, Predator exposure, HPA axis, Post-traumatic stress disorder

\footnotetext{
*Correspondence: pooleyap@msu.edu

${ }^{1}$ Neuroscience Program, Michigan State University, 108 Giltner Hall, 293 Farm

Lane, East Lansing, Ml 48824, USA

Full list of author information is available at the end of the article
}

(c) The Author(s). 2018 Open Access This article is distributed under the terms of the Creative Commons Attribution 4.0 International License (http://creativecommons.org/licenses/by/4.0/), which permits unrestricted use, distribution, and reproduction in any medium, provided you give appropriate credit to the original author(s) and the source, provide a link to the Creative Commons license, and indicate if changes were made. The Creative Commons Public Domain Dedication waiver (http://creativecommons.org/publicdomain/zero/1.0/) applies to the data made available in this article, unless otherwise stated. 


\section{Background}

The neurobiology of sex differences in post-traumatic stress disorder (PTSD) is not understood [1]. The prevalence of PTSD is twice as high in women compared to men, despite an overall lower risk for women to experience a traumatic event $[2,3]$. While men and women experience different types of trauma at different rates, and women have an increased risk for PTSD following assaultive trauma, trauma type alone does not account for this sex difference in PTSD prevalence $[2,4,5]$. This sex difference in PTSD prevalence is evident even when men and women experience the same type of trauma, such as accidents, terrorism, and natural disaster [1]. Furthermore, regardless of trauma type or prevalence, women experience more chronic PTSD and different symptoms and comorbidities than men [3, 6-9]. This evidence suggests sex differences in the underlying neurobiology of the traumatic stress response, yet our current understanding of PTSD is based on males. Recent reviews emphasize the need to examine the neurobiology behind sex differences in PTSD [10], but this call has largely not been met. While the animal literature is replete with reports showing that stress affects males and females differently, and often with opposing effects on behavior, physiology, and the brain $[11,12]$, focus has been on acute or chronic stress as models of anxiety and depression, with little attention given to whether males and females respond differently to traumatic stress.

Two well-validated and commonly used rodent models of PTSD are single prolonged stress (SPS) and predator exposure (PredX). To date, over 200 SPS studies have been published [13], but only one directly compared males and females [14]. Similarly, only a handful of the $>100$ published PredX studies have examined sex differences [15-17]. The SPS model uses physical and chemical stressors (restraint, forced swim, and ether) whereas the PredX model uses exposure to a natural predator (live cat). Exposure (but without direct contact) to a live cat elicits higher proportions of affected rats than predator odor exposure [18], and thus, most closely matches the intensity of the SPS stress paradigm, leading to comparable proportions affected. We chose these two models not only because of the similarities in the apparent impact of the trauma, but also because the stressors used in each model are sufficiently different to allow us to make inferences about the likely generalizability of any sex differences we discover. Fully understanding PTSD requires researchers to directly compare males and females exposed to the same types of stressors under the same conditions, and doing so with two different types of stressors can address whether sex differences in the traumatic stress response are stressor specific or are likely core attributes of traumatic stress in men versus women. Such controlled studies will enable investigators to identify the critical factors contributing to, and thus, the neurobiological underpinnings of, sex-specific responses to traumatic stress. The studies described in this report begin to address this issue.

Presumed core attributes of PTSD include hyper-responsiveness to stressful stimuli, indicated by an enhanced acoustic startle response (ASR) and an exaggerated negative feedback response of the hypothalamic-pituitary-adrenal (HPA) axis revealed by the dexamethasone suppression test under acute stress, leading to chronic hypocortisolism [19]. Such responses to trauma are readily observed in men with PTSD [20] and trauma-exposed male rodents [21]. However, whether these same readouts reflect the effects of trauma in females is much less clear [22]. Indeed, more than half of women with PTSD do not show the male-typical increase in negative feedback control of the HPA axis [23, 24]. Similarly, women with PTSD are less likely to show enhanced ASR, and in some cases, show diminished startle [25]. Current diagnostic criteria and clinical practices have not been revised to reflect these sex differences in the trauma response, largely due to a lack of basic research on females.

We embarked on a series of studies to examine the response of male and female rats exposed to the same traumatic stress, either SPS or PredX. We began by directly comparing intact males to intact, normally cycling females, since sex differences in fear conditioning are present regardless of estrogen levels in women with PTSD [26]. Moreover, sex differences in rat HPA axis activity [27-30] and startle response [31] are not driven by changes in the female hormone cycle. We find robust sex differences in the traumatic stress response at every level of analysis, from behavior to the stress hormone response to cellular measures in the brain. Taken together, these findings begin to uncover novel neurobiological mechanisms underlying sex-specific responses to trauma.

\section{Methods \\ Animals}

Eight-week-old adult Sprague-Dawley male and female rats (total $n=112$ ) were purchased from Charles River (Wilmington, MA, USA) and housed with 12-h reversed light-dark cycle, ad lib food and water. Cage bedding was changed weekly, and no testing was conducted on days of cage changes. Rats were housed in same-sex pairs on the day of arrival and handled 3 min daily for 1 week before any testing or stress exposure. All behavior tests were conducted in the dark phase $\geq 2 \mathrm{~h}$ of dark. Female rats were freely cycling and assigned to treatment groups without regard to estrous cycle stage. All animal procedures and care met or exceeded the NIH guidelines and were approved by Michigan State University Institutional Animal Care and Use Committee. 


\section{Study design}

Male and female rats were exposed to a traumatic stress paradigm or a no-stress control condition, in a $2 \times 2$ design with sex and stress being the two main factors. The experimental timeline (Fig. 1) began with daily handling of rats for 1 week before SPS or PredX. Baseline ASR testing was conducted the day before SPS or PredX, and rats were left undisturbed for 1 week after stress. Post-stress ASR was assessed 8 days later, and dexamethasone (DEX) suppression test (DST) 9 days later. Each experiment was conducted using multiple independent cohorts of rats, with equal numbers of rats representing all treatment groups (minimum of two rats per group) in a single cohort yoked through the entire experiment. The number of cohorts required was determined a priori by a power analysis using effect sizes obtained from preliminary studies to determine the number of rats required per group to achieve 0.80 statistical power, and data from each cohort in a single experiment were collapsed after confirming via ANOVA no significant cohort effects.

\section{Acoustic startle response}

Rats were placed in a Plexiglas tube attached to an accelerometer inside a dark, soundproof chamber (SR-Lab, San Diego Instruments, CA, USA) and allowed to acclimate for $5 \mathrm{~min}$ (68-dB background noise) before delivery of a startle stimulus (50-ms burst of $110-\mathrm{dB}$ white noise every $30 \mathrm{~s}$ for $15 \mathrm{~min}$ ), as previously described [21]. The chamber and Plexiglas tube were cleaned with $70 \%$ ethanol between each test. Peak whole-body startle response was recorded every $1 \mathrm{~ms}$ for $100 \mathrm{~ms}$, beginning with each startle stimulus. The average peak value for each rat was normalized to body weight. Baseline measures were taken on the day before stress exposure, with rats randomly selected and counterbalanced by group and cagemates tested simultaneously in two separate chambers. Rats were then assigned to control or stress groups so that each group had equal average ASR [32]. ASR testing was repeated 8 days after stress exposure with individual rats tested in the same order as baseline ASR. This repeated-measures design is important because inter-individual differences in ASR can be large, but startle response is a stable trait within an individual, both in humans and rats, so any effects of trauma can be detected by comparing changes between preand post-test [33, 34].

\section{SPS paradigm}

All rats were singly housed immediately before exposure to SPS or control conditions to eliminate any sex-specific effects of social support on the stress response. We were particularly concerned that pair housing might mask an effect of trauma in females [35]. SPS consists of a psychological stressor (2-h tube-restraint stress, Braintree Scientific, Braintree, MA, USA), a physiological stressor (20-min group forced swim in $24{ }^{\circ} \mathrm{C}$ water, $n=6$ same-sex rats per 75-l tub with $28 \mathrm{~cm}$ water depth), and a chemical stressor (brief exposure to diethyl ether until immobile and lacking toe-pinch response), as previously described [36]. Rats were given a 15-min rest period in their home cages after the forced swim before ether exposure. Ether is used as a chemical/endocrinological stressor to recapitulate an important phenotypic aspect of PTSD and is not used as an anesthetic. Control rats were also similarly removed from the vivarium for $2.75 \mathrm{~h}$. Rats were left undisturbed for 1 week after SPS, a requisite delay for the long-lasting PTSD responses to develop [21].

\section{PredX paradigm}

Rats were placed in individual wedge-shaped enclosures of a circular Plexiglas "pie restrainer" (Braintree Scientific, Braintree, MA, USA) on which cat food was smeared and placed inside a Plexiglas arena $\left(60 \mathrm{~cm}^{3}\right)$ containing a live

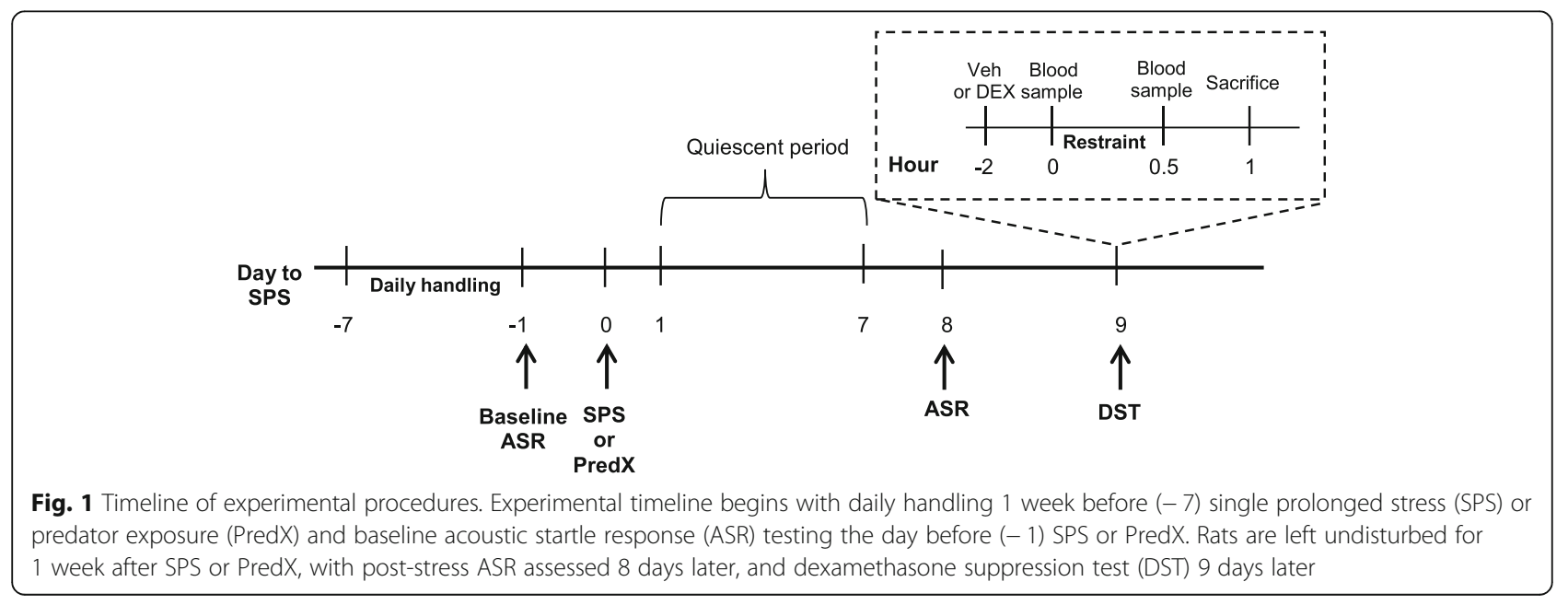


female cat for $1 \mathrm{~h}$, as previously described [16]. This paradigm allows rats to be exposed to the cat without physical contact. All rats were singly housed immediately before the PredX and control procedure. Control rats were removed from the vivarium for $1 \mathrm{~h}$ but not put in the restrainer nor exposed to the cat and were housed in a separate room to prevent exposure to possible residual predator scent on PredX rats. Rats were left undisturbed for 1 week after PredX to allow the acute stress responses to resolve and the long-term responses to trauma develop.

\section{Dexamethasone suppression test (DST)}

The DST is a tool used in clinical and experimental studies to detect disruption of the HPA axis [37]. Dexamethasone (DEX) is a pituitary GR agonist [38] that, when administered hours before a stressful stimulus, diminishes any subsequent cortisol/corticosterone (CORT) response via activation of negative feedback control of the HPA axis. Men with PTSD typically show exaggerated suppression of CORT with DEX compared to healthy controls [39]. To assess the strength of negative feedback control of the HPA axis in response to acute restraint stress, the DST was performed 9 days post-SPS/PredX, as previously described [21]. Rats from each group were randomly assigned to receive either DEX or vehicle. Dexamethasone (Sigma-Aldrich, St. Louis, MO, USA) was dissolved with ethanol and diluted to $5 \%$ in sterile saline. Low-dose DEX $(0.05 \mathrm{mg} / \mathrm{kg}$, i.p.) or vehicle was administered $2 \mathrm{~h}$ prior to 30 min tube-restraint. In rats, this dose of DEX has been determined to produce a submaximal suppression of CORT responses to acute restraint stress that allows for detection of exaggerated DEX suppression of CORT [40]. Tail-nick blood samples were collected at 0 and 30 min of restraint. Blood samples were collected in the rats' dark phase, matching the time of day across experimental groups. Plasma CORT levels were determined using an enzyme immunoassay kit. Rats were overdosed with pentobarbital (i.p.) after $30 \mathrm{~min}$ of restraint, then intracardially perfused with saline and $4 \%$ buffered paraformaldehyde, with brains harvested for staining.

\section{cFos and glucocorticoid receptor (GR)} immunohistochemistry (IHC)

Brains were sectioned and labeled for cFos or GR using a peroxidase $A B C$ kit (Vectastain Elite $A B C$ Kit, Vector Labs, cat\# PK-6200), and only rats that received vehicle injections (and not DEX) were used to map specific neuronal populations activated by acute restraint stress and those expressing GR. A rabbit IgG polyclonal cFos antiserum (1:10,000; Santa Cruz Biotech, cat\# sc-52, Dallas, TX, USA) and diamenobenzidine was used to visualize cFos expression, as previously described [41]. GR IHC used the same basic protocol on alternate sections from the same brains, with a GR primary antiserum (1:2500; rabbit polyclonal IgG; Santa Cruz Biotech, cat\# M-20, Dallas, TX, USA). Specificity of GR staining was confirmed by observing a loss of nuclear staining when the GR antiserum was preadsorbed with the immunizing peptide and observing the expected regional staining (e.g., in the dentate gyrus and CA1 but not CA3). Microscope analysis was conducted on a Zeiss Axioplan light microscope equipped with a video camera and MBF Stereo Investigator software (MBFBioscience, Williston, VT, USA). The number of cFos+ or GR+ neurons within specific brain regions was counted blind from four comparable sections per rat using unbiased stereological methods, and the number of immunopositive cells per cubic millimeter was quantified for each region by dividing the total number of cells counted within a region by the measured volume of that region. Anatomical position was determined using a stereotaxic atlas.

\section{Statistical analysis}

All data were collected by experimenters blind to treatment group. All rats from all cohorts in a single experiment were included in the final statistical analysis, excepting the following exclusion criteria determined a priori: (1) if baseline CORT was not reduced in a DEX-treated rat and experimenter notes confirm a partial/problematic injection, that individual was excluded from only the DST analysis, and (2) if CORT levels did not increase (either 0 or negative change) after $30 \mathrm{~min}$ of restraint, that individual was excluded only from the DST analysis. These exclusion criteria resulted in the exclusion of $0-2$ individuals from each treatment group for the DST. After confirming equal variance between treatment groups, two- or three-way ANOVAs were run for comparisons in rats. See Additional files 1 and 2 for all statistical tests performed. For brain measures, if no main effect or interactions of hemisphere were present, data were collapsed across hemisphere. The conservative Bonferroni test was used to correct for multiple tests to hold the probability of a type I error at 0.05 .

\section{Results}

Male and female rats respond differently to single prolonged stress (SPS)

As expected, males exposed to SPS showed an enhanced acoustic startle response (ASR) (Fig. 2a) compared to their pre-stress baseline ASR $(P=0.017)$, replicating a well-established effect of SPS [21]. On the other hand, SPS had no effect on ASR in females (Fig. 2a). The dexamethasone (DEX) suppression test (DST) also revealed the expected enhanced sensitivity to DEX in SPS-exposed males, blocking the restraint-induced increase in circulating 

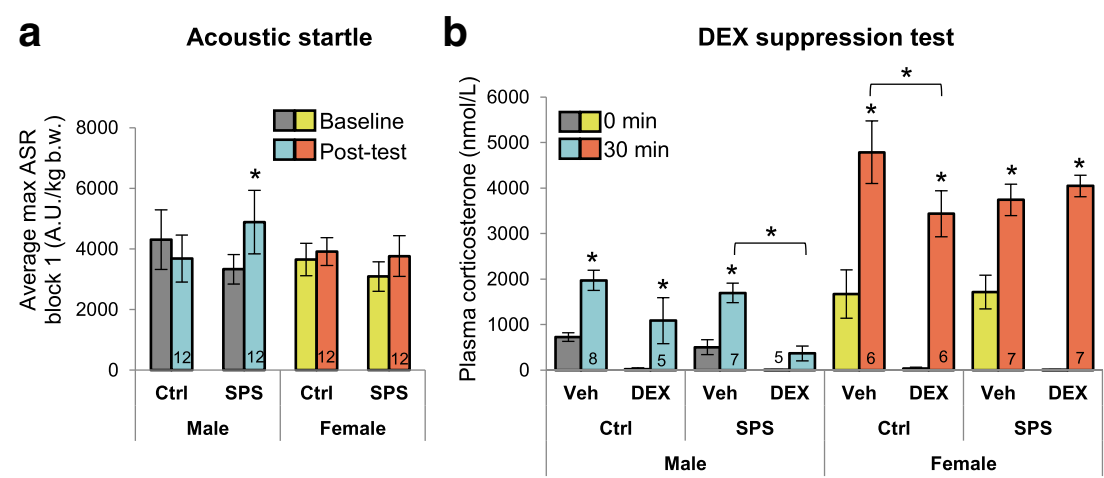

\section{GR expression}
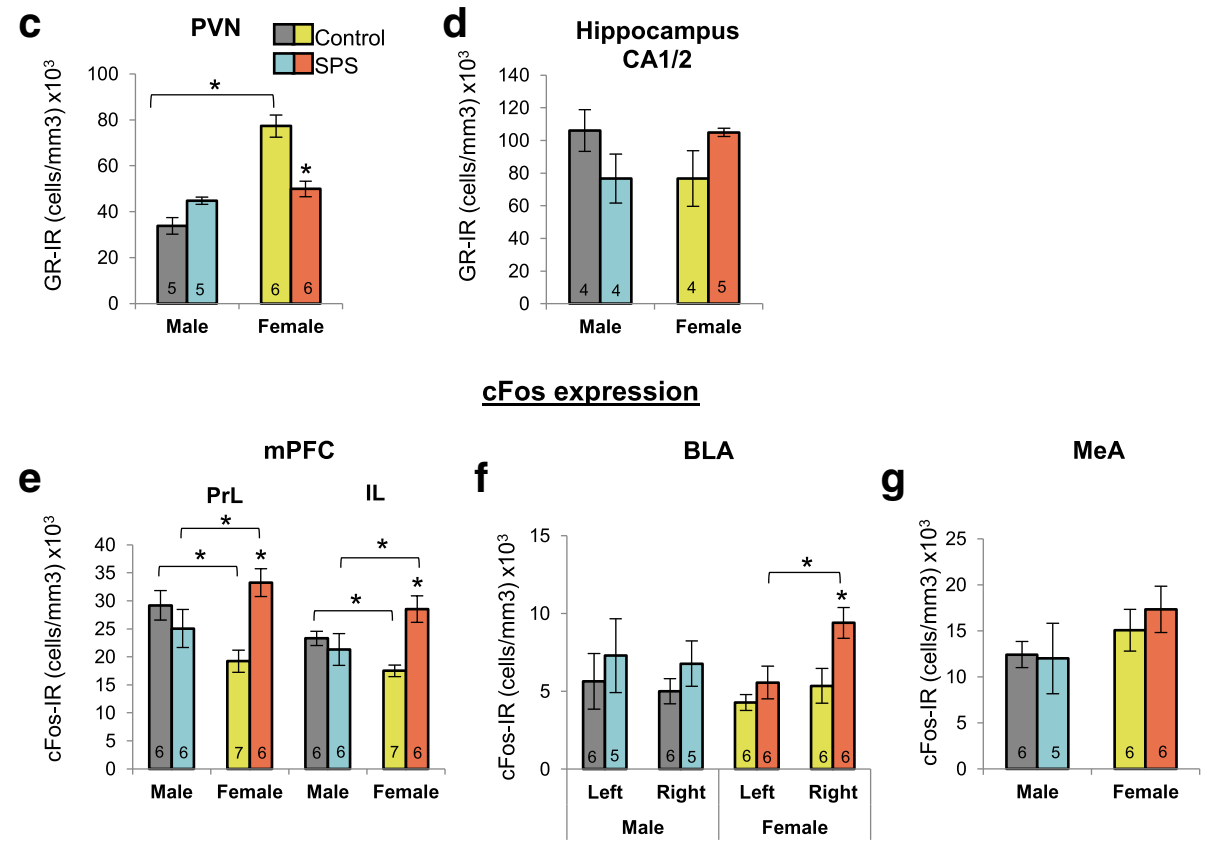

Fig. 2 Single prolonged stress (SPS) affects males and females differently. a Exposure to SPS increased the acoustic startle response (ASR) in male but not in female rats. $\mathbf{b}$ Likewise, the dexamethasone (DEX) suppression test (DST) revealed an enhanced sensitivity to DEX only in SPS-exposed males, with DEX significantly lowering CORT levels after acute restraint stress compared to vehicle for SPS-exposed males. DEX treatment of control males failed to significantly reduce the CORT response. Surprisingly, CORT levels of SPS females after restraint stress were comparable, regardless of DEX treatment, suggesting DEX-nonsuppression, a characteristic of depression. Note that DEX lowered baseline CORT levels (0 min) in all groups, demonstrating its effectiveness in both sexes. CORT levels were significantly higher in females compared to males at both time points, as expected. $\mathbf{c}$, d SPS had divergent effects on glucocorticoid receptor (GR) expression in the brain, with GR expression in the paraventricular nucleus of the hypothalamus (PVN) somewhat increased by SPS in males $(P=.060)$ but significantly decreased in females. SPS also affected GR expression in hippocampal CA1/2, decreasing GR in males but increasing GR in females (sex*SPS interaction $P=.050$ ). GR expression in the PVN of control females was also higher compared to that of control males. e- $\mathbf{g}$ Surprisingly, SPS had no effect on the cFos response of males to restraint stress in the prelimbic (PrL) or infralimbic (IL) subregions of the medial prefrontal cortex (mPFC), basal lateral amygdala (BLA), nor medial amygdala (MeA), but significantly increased cFos responding of females to restraint stress in the PrL, IL, and right BLA of females. The cFos response also showed a sex difference in the $\mathrm{MPFC}$, being lower in control females than control males. Data presented as mean \pm SEM. Significance set at $P<.05$ (indicated by asterisk) for planned pairwise comparisons (Bonferroni). Refer to Additional file 1 for full statistical results

corticosterone (CORT) levels typical of control males (Fig. 2b). In contrast, females exposed to SPS showed a reduced sensitivity-DEX pretreatment, which competes with endogenous CORT for glucocorticoid receptor (GR) binding, failed to suppress the stress-related increase in CORT levels in SPS-exposed females (Fig. 2b). Notice however that for control females, this same dose of DEX effectively suppressed stress-induced increases in CORT levels and that DEX lowered baseline CORT levels $(0 \mathrm{~min})$ in all groups compared to baseline CORT levels in vehicle-treated rats, 
demonstrating its effectiveness in both sexes. In short, these data show the expected exaggerated DEX suppression of CORT in SPS-exposed males but not females. This sex difference in DEX sensitivity clearly reflects the effects of trauma exposure per se, and not resistance to DEX in females, since DEX lowered CORT levels in control females both before and after acute stress but drove only baseline CORT levels down in SPS-exposed females (Fig. 2b). SPS did not affect baseline CORT levels in either sex, and control females showed the expected higher baseline and post-stress CORT levels than males (Fig. 2b).

These striking sex differences in response to SPS suggested that SPS might also differentially affect cellular measures in the brain of males and females. This is indeed what we found when we examined the expression of GR and cFos in brain regions implicated in the control of the traumatic stress response [20]. While SPS moderately increased the number of GR-expressing neurons in the paraventricular nucleus of the hypothalamus (PVN) of males compared to control males $(P=0.06)$, SPS significantly decreased their number in females compared to control females $(P<0.0001$; Fig. $2 \mathrm{c})$. GR expression in the PVN of control (unstressed) females was also higher than that of control males $(P<0.0001$; Fig. 2c). GR expression in the CA1/2 region of the dorsal hippocampus was also affected differentially by SPS in males and females, as SPS decreased the number of GR+ neurons in males compared to control males but increased their number in females compared to control females (Fig. 2d), as previously reported [14], leading to a nearly significant interaction between sex and SPS $(P=0.05)$. SPS had surprisingly little effect on the cFos response to acute restraint stress in the brains of males, including in the prelimbic (PrL) or infralimbic (IL) sugbregions of the medial prefrontal cortex (mPFC) (Fig. 2e), the basolateral amygdala (BLA) and medial amygdala (MeA; Fig. 2f, g). In contrast, SPS broadly affected the cFos response to acute stress in the brains of females, increasing the number of cFos+ neurons more than a week after exposure to trauma in both the PrL $(P=0.001)$ and IL $(P=0.001)$ (Fig. 2e), and in the right BLA $(P=0.021$; Fig. 2f $)$ compared to control females. The number of cFos+ neurons induced by acute stress was also sexually differentiated in the mPFC of control rats, with control females having fewer such neurons than control males (PrL $P=0.011$; IL $P=0.042$; Fig. 2 e), as previously reported [29]. Additionally, body weight cannot account for these sex differences in the trauma response (Table 1) since SPS had no effect on body weight in either sex. Taken together, we found sex differences in the response to SPS in every outcome measure examined. Considering all outcome measures in a correlation matrix (see Additional file 3), there were
Table 1 SPS did not affect body weight in males or females

\begin{tabular}{llll}
\hline Group & & \multicolumn{2}{l}{ Body weight in grams (SEM) } \\
\cline { 3 - 4 } & & Baseline & Post-test \\
\hline Male & Ctrl & $297.2(3.4)$ & $350.0^{*}(7.1)$ \\
& SPS & $297.5(4.9)$ & $341.9^{*}(7.9)$ \\
Female & Ctrl & $191.5(3.0)$ & $213.9^{*}(5.4)$ \\
& SPS & $194.5(3.0)$ & $214.8^{*}(3.2)$
\end{tabular}

Rats were weighed before undergoing SPS or control conditions (baseline) and on the day of the dexamethasone suppression test (post-test). SPS did not affect body weight, and all rats gained weight over the course of the experiment (*, vs baseline). As expected, all females weighed less than males. Data presented as mean \pm SEM. Significance set at $P<.05$ (indicated by asterisk) for planned pairwise comparisons (Bonferroni). Refer to Additional file 1 for full statistical results

significant correlations only between PVN GR and post-stress CORT and between mPFC-IL cFos and right BLA cFos, indicating that most measures were not correlated.

\section{Predator exposure (PredX) induces comparable sex differences in ASR and DST}

To test the generality of this sex difference in the trauma response, we tested adult male and female rats in a different model of PTSD, the PredX model. Rats were exposed to a live female cat for $1 \mathrm{~h}$. Like SPS, PredX enhanced both ASR $(P=0.016$ SPS male post-test compared to baseline; Fig. 3a) and DEX suppression of CORT (Fig. 3b) for males but not females. Specifically, DEX blocked an increase in CORT levels for PredX-exposed males, leading to a significant difference in CORT levels between DEX and vehicle-treated PredX males $(P=0.010)$ but not for control males. Like for SPS, this same trauma-specific effect was not seen in PredX-exposed females, again showing that prior exposure to a traumatic event enhances sensitivity to DEX in males but not in females. Females again displayed the expected higher baseline and post-restraint CORT levels compared to males (Fig. 3b), and DEX lowered baseline CORT levels in all four groups (Fig. 3b), again confirming the effectiveness of the DEX treatment. Females again had higher GR expression in the PVN than males, but unlike SPS, PredX did not affect GR expression in the PVN of either sex (Fig. 3c). Such dissociations in the stress response across different stressors help to identify core traits of the sex-specific phenotype that are independent of stressor type. The effect of trauma on GR expression in the PVN may be stressor specific. Overall, the pattern of sex differences was remarkably similar after exposure to PredX and SPS, and again, occurred independent of effects on body weight (Table 2).

Taken together, these data establish sex-specific responses to traumatic stress that are independent of the type of stressor, showing that the widely presumed core traits of PTSD may only apply to males. 


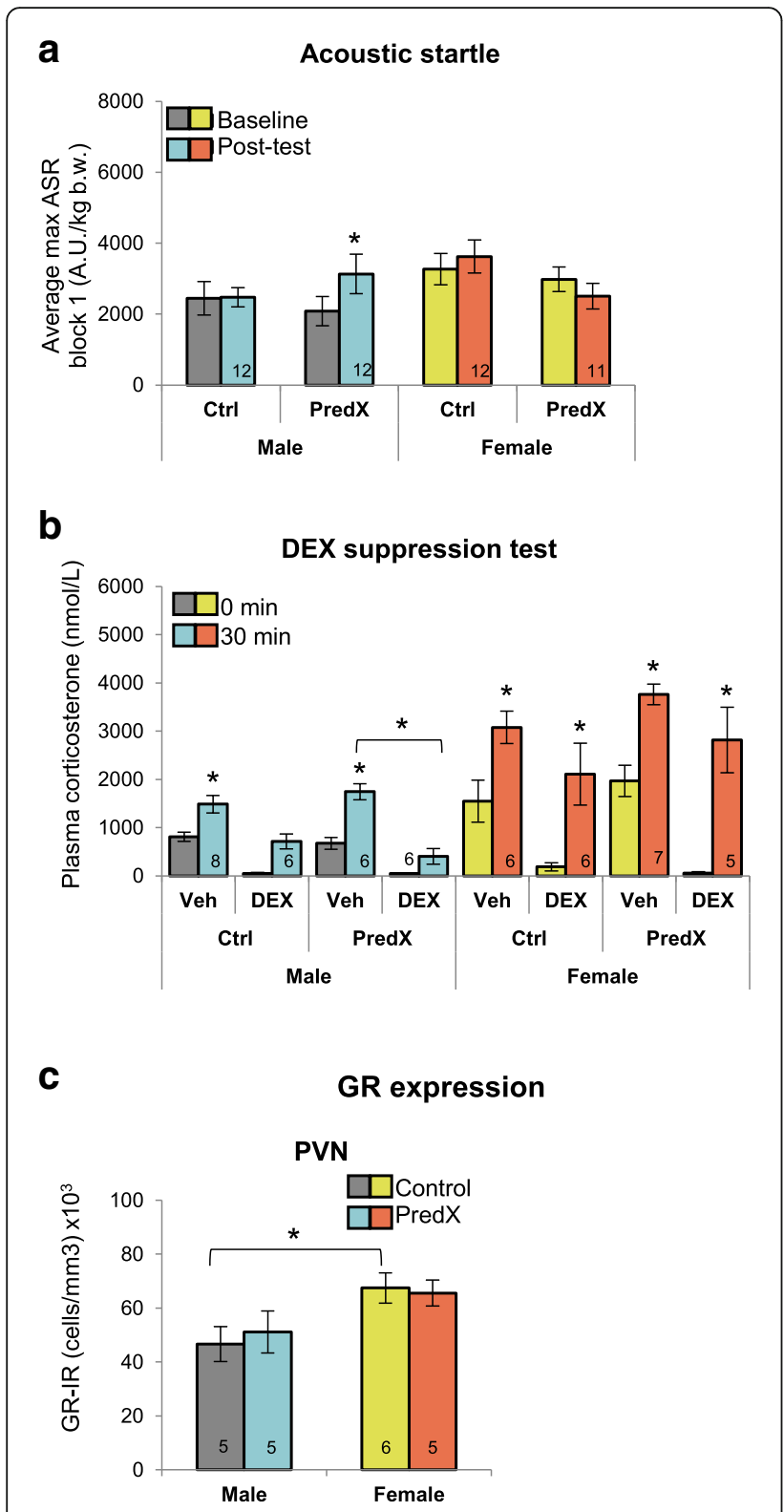

Fig. 3 PredX leads to comparable sex differences in ASR and negative feedback control of CORT. a Only males and not females show an enhanced ASR after PredX exposure, replicating the sex difference found after SPS exposure (Fig. 2a). $\mathbf{b}$ Likewise, PredX enhanced HPA negative feedback in males but not females. DEX blocked the stressinduced increase in CORT levels only in PredX males, indicating an enhanced sensitivity to DEX in this group and not in PredX females, paralleling results in the SPS model (Fig. 2b). As expected, CORT levels were significantly higher in female compared to males. Again, DEX lowered baseline CORT levels (0 min) to near zero in all groups, demonstrating the effectiveness of DEX in both sexes. c Unlike SPS, PredX did not affect GR expression in the PVN of either sex, although the baseline sex difference was replicated (see Fig. 2c); females have more GR+ neurons in the PVN than males. These data suggest that GR expression in the PVN may be responsive to only some types of stress. Data are presented as mean \pm SEM. Significance set at $P<.05$ (indicated by asterisk) for planned pairwise comparisons (Bonferroni). Refer to Additional file 2 for full statistical results
Table 2 PredX did not affect body weight in males or females

\begin{tabular}{llll}
\hline Group & & \multicolumn{2}{l}{ Body weight in grams (SEM) } \\
\cline { 3 - 4 } & & Baseline & Post-test \\
\hline Male & Ctrl & $277.1(3.8)$ & $314.5^{*}(5.2)$ \\
& PredX & $273.2(3.9)$ & $306.2^{*}(4.8)$ \\
Female & Ctrl & $175.8(2.3)$ & $191.9^{*}(2.4)$ \\
& PredX & $176.1(1.7)$ & $192.9^{*}(2.0)$ \\
\hline
\end{tabular}

Rats were weighed before undergoing PredX or control conditions (baseline) and on the day of the dexamethasone suppression test (post-test). PredX did not affect body weight and all rats gained weight over the course of the experiment $\left({ }^{*}\right.$, vs baseline). As expected, all females weighed less than males. Data presented as mean \pm SEM. Significance set at $P<.05$ (indicated by asterisk) for planned pairwise comparisons (Bonferroni). Refer to Additional file 2 for full statistical results

Such data also indicate that there are fundamental sex differences in the neurobiology underlying the traumatic stress response.

\section{Discussion}

Sex differences in the traumatic stress response are among the most widely reported phenomena in epidemiological and clinical studies, but the neurobiological basis for these differences is unknown, largely due to an overwhelming male bias in preclinical research [22]. We find robust and novel sex differences in the traumatic stress response. Only male rats showed a hyper-responsive phenotype with an enhanced ASR and exaggerated negative feedback control of the stress hormone response, both core attributes of PTSD in men [21, 39]. SPS did not affect baseline CORT in either sex, as previously reported for males [36], further suggesting that the effect of trauma is on the negative feedback control of the HPA axis in males. These same measures suggest no effect of trauma in females, but brain measures indicated otherwise.

For example, we found that SPS exposure nearly 2 weeks earlier increased cFos activity in the MPFC and in the right BLA of females but not males. Based on lesion and imaging studies, the right amygdala is uniquely implicated in contextual fear conditioning in rats [42] and humans with PTSD [43, 44] such that the right amygdala is dominant over the left amygdala in emotional processing and fear learning. In humans with PTSD, symptom severity is positively correlated with regional cerebral blood flow to the right, but not left, amygdala $[45,46]$. Whether this amygdalar lateralization is due to lateralized neurocircuitry and/or neurochemical differences is not yet known. Neural projections from the mPFC to the BLA, which are involved in conditioned fear responses, are thought to be disrupted in PTSD and are differentially recruited in male and female rats during fear conditioning and extinction [12]. Thus, our findings of female-specific activation in the MPFC and BLA during acute restraint stress that reminds females of 
a prior trauma experience could reflect these sex-specific mechanisms of fear conditioning and extinction.

We also observed diametrically opposed effects of trauma on GR expression in the PVN and CA1/2 region of the dorsal hippocampus of males and females (Fig. 2c, d), conforming to the opposing effects of stress in males and females in other rodent studies [47-50]. In response to SPS, we found that females exhibited increased GR expression in the CA $1 / 2$ region of the dorsal hippocampus while males showed the opposite. These results corroborate the only other study examining sex differences in this model, which found that these sex differences in GR expression correlated with sex differences in fear extinction retention [14]. The sex-specific response of decreased GR expression in the female PVN likely alters the sensitivity of the PVN to stress hormones and may explain why trauma-exposed males show enhanced negative feedback control of the HPA axis, while trauma-exposed females do not. However, changes in GR in the PVN cannot be the only factor contributing to the divergent effects of trauma on negative feedback control of the CORT response since PredX had no effect on GR expression in the PVN of either sex but had the same divergent effects on the HPA axis. Nonetheless, PTSD patients have an increased number of GR-expressing blood lymphocytes and increased sensitivity to glucocorticoids [51, 52], suggesting that measures of GR expression in blood may well reflect the effects of traumatic stress on GR expression in the brain; however, it should be noted that these populations included predominately males and any sex-specific effects of traumatic stress on GR have not been investigated.

While it seems that the same neural correlates (e.g., hippocampus, mPFC, and amygdala) are enlisted to manage stress in males and females, the specific mechanisms involved are fundamentally different $[12,53]$. The robust sex differences found in some measures of control rats (e.g., females have higher baseline and post-stress CORT levels, higher GR expression in the PVN, and lower cFos expression in the MPFC after acute restraint) have been previously reported and may well predispose males and females to respond differently to traumatic stress [29, 30, 54, 55].

A recent study indicates that trauma does not in general affect the cFos response of the mPFC and amygdala of males [56], consistent with our own results. Interestingly, however, this study identified a small minority (15\%) of males that responded to trauma with increased cFos expression in both IL mPFC and BLA, similar to what we see in females exposed to SPS (Fig. 2e, f), and this cFos response was associated with anhedonic behavior [56]. These data suggest mPFC and amygdala activation may be relevant markers of trauma pathology for females but generally not males and may be associated with an anhedonic behavioral phenotype in females. Future studies would benefit by including behavioral measures of anhedonia that might better illuminate the nature of the sex difference in the traumatic stress response. Indeed, a depressive-like phenotype in SPS-exposed females is suggested by the decreased sensitivity to DEX and decreased GR expression in the PVN we report here, as depressed patients show decreased DEX sensitivity and decreased lymphocyte GR expression [51]. Taken together, the implication of our findings is that the trauma response in females may share common attributes of depression, characterized by increased MPFC and amygdala activity, blunted ASR [57], and reduced rather than increased HPA negative feedback $[39,58]$. Indeed, the traumatic stress response in humans is sex-biased, as men with PTSD show more externalizing symptoms (e.g., hyperarousal, aggression, and risk-taking behaviors), while women with PTSD show more internalizing symptoms (e.g., sadness, loss of pleasure, and social difficulties) [7, 9, 59, 60]. We also recently discovered sex-specific effects of SPS on sucrose preference and social interaction, common measures of depressive-like behavior, only in female rats and not in males (see companion paper), further implicating a depressive-like phenotype of the female trauma response. These results suggest an important consideration: if only outcome measures developed in males are used to detect the effects of traumatic stress in females, erroneous conclusions will likely be drawn.

Indeed, based only on the ASR and DST, females might appear more resilient than males to the deleterious effects of traumatic stress, but as we looked further, it became clear that females are not necessarily more resilient but rather, respond differently to trauma. This is reminiscent of other effects of stress on males and females. For example, in response to uncontrollable footshocks, females appear more resilient than males to the negative effects of stress on learning that involves operant conditioning [61], but if classical conditioning is involved, females are less resilient than males [62], suggesting that sex may not be a factor that confers susceptibility or resilience to stress per se but simply leads to different responses. Clearly, the choice of outcome measures can determine the conclusions drawn. Our data suggest that the line between resiliency and susceptibility is not as clear as presumed [63], largely because whether an individual is judged as susceptible is tied to the measures used (Additional file 4). Moreover, an individual may be resilient in one domain of functioning but not in another, or at one time during the lifespan but not another [64]. Put more simply, female rats in these studies can be said to be resilient to trauma only if one starts with the presumption that only male-typical responses to trauma are valid. 
While our data seem to question the heuristic value of the terms "resilience" and "susceptibility," these terms remain useful and valid. Not only does the epidemiological data tell us that only a small fraction of people exposed to trauma develop PTSD, indicating that differences in susceptibility are real, but also a growing list of identified gene polymorphisms and epigenetic states appear to bias the nervous system toward more or less resilience against the negative effects of stress. Nonetheless, our data suggest that the use of these terms may be defined differently when sex is considered.

\section{Conclusions}

In sum, we are the first to describe distinct responses to traumatic stress in two different animal models linked to a single biological factor: sex. We propose that such sex differences reflect differences in the underlying neurobiology. This conclusion has wide reaching implications for therapies that will effectively treat PTSD in both men and women. Factors that mediate differences in how individuals adjust after traumatic stress are attractive targets for the prevention and treatment of PTSD. At least $75 \%$ of people in the USA experience a traumatic event in their lifetime and do not develop PTSD [65], begging the question of why some people who experience trauma develop PTSD while others do not? The significant sex differences in the prevalence of stress-related disorders in humans is a call for inquiries into the factors behind such differences, which will undoubtedly offer insight into the brain regions and mechanisms that underlie individual differences in the response to traumatic stress.

\section{Additional files}

Additional file 1: Statistical results for data shown in Fig. 2 and Table 1. All pairwise comparisons use Bonferroni adjustment for multiple comparisons. RM denotes repeated measure; otherwise assume between group measures. Only statistically significant pairwise comparisons are shown. (DOCX $25 \mathrm{~kb}$ )

Additional file 2: Statistical results for data shown in Fig. 3 and Table 2. All pairwise comparisons use Bonferroni adjustment for multiple comparisons. RM denotes repeated measure; otherwise assume between group measures. Only statistically significant pairwise comparisons are shown. (DOCX $21 \mathrm{~kb})$

Additional file 3: Descriptive statistics and correlation matrix for SPS study variables. ${ }^{* *}$ Correlation is significant at the 0.01 level (2-tailed); ${ }^{*}$ Correlation is significant at the 0.05 level (2-tailed). (DOCX $17 \mathrm{~kb}$ )

Additional file 4: Susceptibility and resilience are a function of the measure considered. Even within males, different measures of traumatic stress indicate different degrees of vulnerabilities to traumatic stress within a given individual. Note that for one SPS-exposed male (red triangle), the acoustic startle response (ASR) is markedly elevated indicating a severe effect of SPS on this male. However, based on the dexamethasone (DEX) suppression test (DST), this same male is minimally affected by SPS. Moreover, glucocorticoid receptor (GR) expression in the paraventricular nucleus of the hypothalamus (PVN) indicates that SPS had a moderate effect on this same male. Other individual males exposed to SPS also show marked variability across these three measures, underscoring the idea that like many psychological phenomena, accurate diagnosis of PTSD requires a composite view of numerous diagnostic measures. Values for all individuals are indicated by the colored circles, with the mean (black larger circle) and standard deviation (black error bars) of these values. (DOCX $112 \mathrm{~kb}$ )

\section{Abbreviations}

ASR: Acoustic startle response; BLA: Basolateral amygdala; CORT: Corticosterone; DEX: Dexamethasone; DST: Dexamethasone suppression test; GR: Glucocorticoid receptor; HPA: Hypothalamic-pituitaryadrenal; IHC: Immunohistochemistry; IL: Infralimbic region of the medial prefrontal cortex; MeA: Medial amygdala; mPFC: Medial prefrontal cortex; PredX: Predator exposure; PrL: Prelimbic region of the medial prefrontal cortex; PTSD: Post-traumatic stress disorder; PVN: Paraventricular nucleus of the hypothalamus; SPS: Single prolonged stress

\section{Acknowledgements}

The authors thank Diane Redenius and Kate Mills for the technical support.

\section{Funding}

This work was funded by grants MH111604 (NIMH) to A.J.R., DA039895 (NIDA) to M.S.M., R21MH104780 (NIH) to S.M.B., and R01NS045195 (NIH) and Cohen Veteran Bioscience to C.L.J.

\section{Availability of data and materials}

All data are available from the corresponding author upon request.

\section{Authors' contributions}

AEP conceived the research question and strategy, performed all research, analyzed data, and wrote the paper. RCB designed and performed research for the PredX study. SS designed and performed research for the female housing study. ALE, AJR, MSM, and SMB gave technical support and conceptual advice. CLJ gave technical support and conceptual advice and wrote the paper. All authors discussed the results and implications and commented on the manuscript at all stages. All authors read and approved the final manuscript

\section{Ethics approval and consent to participate}

All animal procedures and care met or exceeded the $\mathrm{NIH}$ guidelines and were approved by Michigan State University Institutional Animal Care and Use Committee.

\section{Consent for publication}

Not applicable

\section{Competing interests}

The authors declare that they have no competing interests.

\section{Publisher's Note}

Springer Nature remains neutral with regard to jurisdictional claims in published maps and institutional affiliations.

\section{Author details}

${ }^{1}$ Neuroscience Program, Michigan State University, 108 Giltner Hall, 293 Farm Lane, East Lansing, MI 48824, USA. ²Department of Physiology, Michigan State University, 2201 BPS, 567 Wilson Rd, East Lansing, MI 48824, USA.

Received: 6 April 2018 Accepted: 26 June 2018

Published online: 05 July 2018

References

1. Tolin DF, Foa EB. Sex differences in trauma and posttraumatic stress disorder: a quantitative review of 25 years of research. Psychol Bull. 2006; 132:959-92.

2. Kessler RC, Sonnega A, Bromet E, Hughes M, Nelson C. Posstraumatic stress disorder in the national comorbidity survey. Arch Gen Psychiatry. 1995;52:1048-60.

3. Breslau N. Gender differences in trauma and posttraumatic stress disorder. J Gend Specif Med. 2002;5:34-40. 
4. Breslau N, Anthony JC. Gender differences in the sensitivity to posttraumatic stress disorder: an epidemiological study of urban young adults. J Abnorm Psychol. 2007;116:607-11.

5. Breslau N, Davis GC, Andreski P, Peterson EL, Schultz LR. Sex differences in posttraumatic stress disorder. Arch Gen Psychiatry. 1997;54:1044-8.

6. King MW, Street A, Gradus JL, Vogt DS, Resick PA. Gender differences in posttraumatic stress symptoms among OEF/OIF veterans: an item response theory analysis. J Trauma Stress. 2013;26:175-83.

7. Castillo DT, Joseph JS, Tharp AT, De BJC, Torres-sena LM, Qualls C, et al. Externalizing and internalizing subtypes of posttraumatic psychopathology and anger expression. J Trauma Stress. 2014;27:108-11.

8. Zlotnick C, Zimmerman M, Wolfsdorf BA, Mattia Jl. Gender differences in patients with posttraumatic stress disorder in a general psychiatric practice. Am J Psychiatry. 2001;158:1923-5.

9. Rielage JK, Hoyt T, Renshaw K. Internalizing and externalizing personality styles and psychopathology in OEF-OIF veterans. J Trauma Stress. 2010;23:350-7.

10. Cohen H, Kozlovsky N, Alona C, Matar MA, Joseph Z. Animal model for PTSD: from clinical concept to translational research. Neuropharmacology Elsevier Ltd. 2012;62:715-24.

11. Farrell MR, Sengelaub DR, Wellman CL. Sex differences and chronic stress effects on the neural circuitry underlying fear conditioning and extinction. Physiol Behav Elsevier Inc. 2013;122:208-15.

12. Gruene TM, Roberts E, Thomas V, Ronzio A, Shansky RM. Sex-specific neuroanatomical correlates of fear expression in prefrontal-amygdala circuits. Biol Psychiatry Elsevier. 2015;78:186-93.

13. Souza RR, Noble LJ, Mclntyre CK. Using the single prolonged stress model to examine the pathophysiology of PTSD. Front Pharmacol. 2017;8:1-9.

14. Keller SM, Schreiber WB, Staib JM, Knox D. Sex differences in the single prolonged stress model. Behav Brain Res Elsevier BV. 2015;286:29-32.

15. Koresh O, Kaplan Z, Zohar J, Matar MA, Geva AB, Cohen H. Distinctive cardiac autonomic dysfunction following stress exposure in both sexes in an animal model of PTSD. Behav Brain Res. Elsevier B.V. 2016;308:128-42.

16. Burke HM, Robinson CM, Wentz B, McKay J, Dexter KW, Pisansky JM, et al. Sex-specific impairment of spatial memory in rats following a reminder of predator stress. Stress. 2013;16:469-76.

17. Daskalakis NP, Cohen H, Cai G, Buxbaum JD, Yehuda R. Expression profiling associates blood and brain glucocorticoid receptor signaling with trauma-related individual differences in both sexes. Proc Natl Acad Sci. 2014;111:1-6.

18. Cohen H, Zohar J, Matar MA, Zeev K, Loewenthal U, Richter-Levin G. Setting apart the affected: the use of behavioral criteria in animal models of post traumatic stress disorder. Neuropsychopharmacology. 2004;29:1962-70.

19. Sherin JE, Nemeroff CB. Post-traumatic stress disorder: the neurobiological impact of psychological trauma. Dialogues Clin Neurosci. 2011;13:263-78.

20. Pitman RK, Rasmusson AM, Koenen KC, Shin LM, Orr SP, Gilbertson MW, et al. Biological studies of post-traumatic stress disorder. Nat Rev Neurosci Nature Publishing Group. 2012;13:769-87.

21. Kohda K, Harada K, Kato K, Hoshino A, Motohashi J, Yamaji T, et al. Glucocorticoid receptor activation is involved in producing abnormal phenotypes of single-prolonged stress rats: a putative post-traumatic stress disorder model. Neuroscience. 2007;148:22-33.

22. Shansky RM. Sex differences in PTSD resilience and susceptibility: challenges for animal models of fear learning. Neurobiol Stress. Elsevier Inc. 2015;1:60-5.

23. Metzger $\amalg$, Carson MA, Lasko NB, Paulus LA, Orr SP, Pitman RK, et al. Basal and suppressed salivary cortisol in female Vietnam nurse veterans with and without PTSD. Psychiatry Interpers Biol Process. 2008;161:330-5.

24. Zaba M, Kirmeier T, Ionescu IA, Wollweber B, Buell DR, Gall-Kleebach DJ, et al. Identification and characterization of HPA-axis reactivity endophenotypes in a cohort of female PTSD patients. Psychoneuroendocrinology. Elsevier Ltd; 2015;55:102-115.

25. Medina AM, Mejia VY, Schell a M, Dawson ME, Margolin G. Startle reactivity and PTSD symptoms in a community sample of women. Psychiatry Res. 2001;101:157-69.

26. Shvil E, Sullivan GM, Schafer S, Markowitz JC, Campeas M, Wager TD, et al. Sex differences in extinction recall in posttraumatic stress disorder: a pilot fMRI study. Neurobiol Learn Mem Elsevier Inc. 2014;113:101-8.

27. Handa RJ, Burgess L, Kerr J, O'Keefe J. Gonadal steroid hormone receptors and sex differences in the hypothalamic-pituitary-adrenal axis. Horm Behav. 1994;28:464-76.
28. Spinedi E, Suescun MO, Hadid R, Gaillard RC. Effects of gonadectomy and sex hormone therapy on the endotoxin-stimulated hypothalamo-pituitaryadrenal axis: evidence for a neuroendocrine-immunological sexual dimorphism EDUARDO. Endocrinology. 1992;131:2430-6.

29. Bland ST, Schmid MJ, Der-Avakian A, Watkins LR, Spencer RL, Maier SF. Expression of c-fos and BDNF mRNA in subregions of the prefrontal cortex of male and female rats after acute uncontrollable stress. Brain Res. 2005; 1051:90-9.

30. Babb JA, Masini CV, Day HEW, Campeau S. Sex differences in activated corticotropin-releasing factor neurons within stress-related neurocircuitry and hypothalamic-pituitary-adrenocortical axis hormones following restraint in rats. Neuroscience. 2013;234:40-52.

31. Mazor A, Matar MA, Kaplan Z, Kozlovsky N, Zohar J, Cohen H. Genderrelated qualitative differences in baseline and post-stress anxiety responses are not reflected in the incidence of criterion-based PTSD-like behaviour patterns. World J Biol Psychiatry. 2009;10:856-69.

32. Jia M, Smerin SE, Zhang L, Xing G, Li X, Benedek D, et al. Corticosterone mitigates the stress response in an animal model of PTSD. J Psychiatr Res. 2014;60:29-39.

33. Geyer M a, Swerdlow NR. Measurement of startle response, prepulse inhibition, and habituation. Curr Protoc Neurosci. 2001;Chapter 8:Unit 8.7.

34. Grillon C, Morgan CA, Southwick SM, Davis M, Charney DS. Baseline startle amplitude and prepulse inhibition in Vietnam veterans with posttraumatic stress disorder. Psychiatry Res. 1996;64:169-78.

35. Westenbroek C, Ter Horst GJ, Roos MH, Kuipers SD, Trentani A, den Boer JA. Gender-specific effects of social housing in rats after chronic mild stress exposure. Prog Neuro-Psychopharmacol Biol Psychiatry. 2003;27:21-30.

36. Liberzon I, Krstov M, Young EA. Stress-restress: effects on ACTH and fast feedback. Psychoneuroendocrinology. 1997;22:443-53.

37. Fountoulakis K, lacovides A, Fotiou F, Karamouzis M, Demetriadou A, Kaprinis G. Relationship among dexamethasone suppression test, personality disorders and stressful life events in clinical subtypes of major depression: an exploratory study. Ann Gen Hosp Psychiatry. 2004;3:15.

38. Cole MA, Kim PJ, Kalman BA, Spencer RL. Dexamethasone suppression of corticosteroid secretion: evaluation of the site of action by receptor measures and functional studies. Psychoneuroendocrinology. 2000;25:151-67.

39. Yehuda R. Post-traumatic stress disorder. N Engl J Med. 2002;346:108-14.

40. Jaferi A, Nowak N, Bhatnagar S. Negative feedback functions in chronically stressed rats: role of the posterior paraventricular thalamus. Physiol Behav. 2003;78:365-73.

41. Zuloaga DG, Poort JE, Jordan CL, Breedlove SM. Male rats with the testicular feminization mutation of the androgen receptor display elevated anxietyrelated behavior and corticosterone response to mild stress. Horm Behav Elsevier Inc. 2011;60:380-8.

42. Baker KB, Kim JJ. Amygdalar lateralization in fear conditioning: evidence for greater involvement of the right amygdala. Behav Neurosci. 2004;118:15-23.

43. Engdahl B, Leuthold AC, Tan H-RM, Lewis SM, Winskowski AM, Dikel TN, et al. Post-traumatic stress disorder: a right temporal lobe syndrome? J Neural Eng. 2010;7:066005.

44. Bremner JD, Vermetten E, Vythilingam M, Afzal N, Schmahl C, Elzinga B, et al. Neural correlates of the classic color and emotional stroop in women with abuse-related posttraumatic stress disorder. Biol Psychiatry. 2004;55:612-20

45. Shin LM, Orr SP, Carson MA, Rauch SL, Macklin ML, Lasko NB, et al. Regional cerebral blood flow in the amygdala and medial prefrontal cortex during traumatic imagery in male and female Vietnam veterans with PTSD. Arch Gen Psychiatry. 2004;61:168-76.

46. Pissiota A, Frans O, Fernandez M, von Knorring L, Fischer H, Fredrikson M. Neurofunctional correlates of posttraumatic stress disorder: a PET symptom provocation study. Eur Arch Psychiatry Clin Neurosci. 2002;252:68-75.

47. Maeng LY, Milad MR. Sex differences in anxiety disorders: interactions between fear, stress, and gonadal hormones. Horm Behav Elsevier BV. 2015;76:106-17.

48. Shors TJ. A trip down memory lane about sex differences in the brain. Philos Trans R Soc B Biol Sci. 2016;371:20150124.

49. Shors TJ, Beylin AV, Wood GE, Gould E. The modulation of Pavlovian memory. Behav Brain Res. 2000;110:39-52.

50. Wood GE, Shors TJ. Stress facilitates classical conditioning in males, but impairs classical conditioning in females through activational effects of ovarian hormones. Proc Natl Acad Sci. 1998;95:4066-71. 
51. Yehuda R, Boisoneau D, Mason JW, Giller EL. Glucocorticoid receptor number and cortisol excretion in mood, anxiety, and psychotic disorders. Biol Psychiatry. 1993;34:18-25.

52. Rohleder N, Joksimovic L, Wolf JM, Kirschbaum C. Hypocortisolism and increased glucocorticoid sensitivity of pro-inflammatory cytokine production in Bosnian war refugees with posttraumatic stress disorder. Biol Psychiatry. 2004;55:745-51.

53. Wood GE, Beylin AV, Shors TJ. The contribution of adrenal and reproductive hormones to the opposing effects of stress on trace conditioning in males versus females. Behav Neurosci. 2001;115:175-87.

54. Zavala JK, Fernandez AA, Gosselink KL. Female responses to acute and repeated restraint stress differ from those in males. Physiol Behav. Elsevier Inc. 2011;104:215-21.

55. Seale JV, Wood SA, Atkinson HC, Bate E, Lightman SL, Ingram CD, et al. Gonadectomy reverses the sexually diergic patterns of circadian and stressinduced hypothalamic-pituitary-adrenal axis activity in male and female rats. J Neuroendocrinol. 2004;16:516-24.

56. Ritov G, Boltyansky B, Richter-Levin G. A novel approach to PTSD modeling in rats reveals alternating patterns of limbic activity in different types of stress reaction. Mol psychiatry. Nat Publ Group. 2016;21:630-41.

57. Mcteague LM, Lang PJ, Laplante M, Cuthbert BN, Shumen JR, Bradley MM. Aversive imagery in posttraumatic stress disorder: trauma recurrence, comorbidity, and physiological. Biol psychiatry. Elsevier Inc. 2010;67:346-56.

58. Jokinen J, Nordström P. HPA axis hyperactivity and attempted suicide in young adult mood disorder inpatients. J Affect Disord Elsevier BV. 2009:116:117-20.

59. Wolf EJ, Miller MW, Harrington KM, Reardon A. Personality-based latent classes of posttraumatic psychopathology: personality disorders and the internalizing/externalizing model. J Abnorm Psychol. 2012;121:256-62.

60. Miller MW, Resick PA. Internalizing and externalizing subtypes in female sexual assault survivors: implications for the understanding of complex PTSD. Behav Ther. 2007;38:58-71.

61. Shors TJ, Mathew J, Sisti HM, Edgecomb C, Beckoff S, Dalla C. Neurogenesis and helplessness are mediated by controllability in males but not in females. Biol Psychiatry. 2007;62:487-95.

62. Leuner B, Mendolia-Loffredo S, Shors TJ. Males and females respond differently to controllability and antidepressant treatment. Biol Psychiatry. 2004;56:964-70.

63. Nasca C, Bigio B, Zelli D, Nicoletti F, McEwen BS. Mind the gap: glucocorticoids modulate hippocampal glutamate tone underlying individual differences in stress susceptibility. Mol Psychiatry Nature Publishing Group. 2015;20:755-63.

64. Southwick SM, Bonanno GA, Masten AS, Panter-brick C, Yehuda R. Resilience definitions, theory, and challenges: interdisciplinary perspectives. Eur J Psychotraumatol. 2014;5:1-14.

65. Sledjeski EM, Speisman B, Dierker LC. Does number of lifetime traumas explain the relationship between PTSD and chronic medical conditions? Answers from the National Comorbidity Survey-Replication (NCS-R). J Behav Med. 2008;31:341-9.

\section{Ready to submit your research? Choose BMC and benefit from:}

- fast, convenient online submission

- thorough peer review by experienced researchers in your field

- rapid publication on acceptance

- support for research data, including large and complex data types

- gold Open Access which fosters wider collaboration and increased citations

- maximum visibility for your research: over $100 \mathrm{M}$ website views per year

At BMC, research is always in progress.

Learn more biomedcentral.com/submissions 